\title{
RFID and Pose Invariant Face Recognition Based Automated Security System
}

\author{
Tamil Vani .R ${ }^{1}$, Ruckmani Divakaran ${ }^{2}$, Vinitha Shree .G.R ${ }^{3}$, Revathi . $\mathbf{R}^{3}$, Nalini .P. ${ }^{3}$, Khutija Kubra ${ }^{3}$ \\ Assistant Professor, Dr. TTIT, K.G.F ${ }^{1}$ \\ HOD of ECE, Dr. TTIT, K.G.F ${ }^{2}$ \\ Students, Dr. TTIT, K.G.F ${ }^{3}$
}

\begin{abstract}
Conventional methods of using Barcodes that requires line of sight, pricing discrepancies, scanning problems, label damage, financial and equipment cost causes inaccuracy in entering the information and barcode scanners eventually breakdown causing scanning problems. Therefore, Radio Frequency Identification (RFID) technology is proposed that uses radio waves to transfer from an electronic tag called RFID tag, attached to an object, through a reader for the purpose of identifying and tracking the object. RFID technology which is a matured technology that has been widely deployed by various organization as a part of their automation system. In this study, an RFID based system has built in order to produce a compact and reliable smart security system using RFID and face verification is presented here. The RFID system identifies the student using the RFID card and further identity verification of the student is carried out using face recognition technique. RFID uniquely identifies the student based on the card number, then Viola- Jones algorithm is used to verify face of the students using face image of the student. The performance of the system is carried out with RFID code and face recognition.
\end{abstract}

Keywords: RFID card, RFID reader, Viola-Jones Algorithm.

\section{INTRODUCTION}

The most common method of tracking entry of students in the college is the ID cards which have to be inspected manually, for a college of larger strength, this method is cumbersome and time consuming. Hence, the current traditions of inspecting ID cards manually are unreliable and students effortlessly counterfeit the system. Other reliable methods which are sparsely used because of the cost of implementation include the fingerprint recognition system. They offer reliable performance but there are three common issues like false acceptances, false rejections and change in sizes or form/pattern over time. Recently Radio-frequency identification (RFID) based system is significantly used in all the institutions. They are useful for identifying the student but it cannot reliably verify the student who is using it. A student can easily mark bogus attendance for his friend using his card. Then, a secret pin along with RFID is used to verify the student but they too could be easily shared or lost by the students. Hence Face recognition with RFID system used the photograph to identify and verify the faces of the individual student. This approach suggests that the face recognition is viable biometric identification of the future. A compact and reliable automated college gate control system using face verification and RFID is presented in this proposed project. The RFID system identifies the student using the RFID card and further identity verification of the student is carried out using face recognition technique. RFID Uniquely identifies the student based on the card number. The performance of the system is tested for frontal face verification. The schematic diagram of the proposed scheme is shown in block diagram. The system is designed to first identify the student and verify the respective student biometrically. It increases the security and accuracy because of two levels of authentication. RFID Systems has evidences to be the smartest way for student identification. Several biometric verification methods exist like Iris, Face, Fingerprint, etc. Face verification robust method of biometric verification. RFID System uses the RFID cards to identify the student. Face Verification System uses an individual verify the face of each student exclusively. Once the student is identified and is verified the gate is opened. If RFID Card is unidentified by RFID System then it is rejected reporting unrecognized student. If RFID card identification succeeds and face verification failed then proxy person is detected and hence identity of fraud student is known. Thus, the system is designed to be fool-proof against proxy college entry. The system is flexible enough to suit to different conditions by just sending an SMS to alter the working of it. The face verification can be deactivated in cases of functions and large gatherings. RFID student identification system here, every student is provided with an RFID card. RFID cards are assigned a unique 10 digit RFID card numbers by the manufacturer. The card number is only readable and therefore it is mapped to their respective student USN number in a RFID database. Low frequency $(125 \mathrm{KHz})$ RFID card is used which could only be detected at a range from the RFID reader. Thus, collisions of RFID0 cards are avoided. Smart RFID proximity contactless card Reader $125 \mathrm{KHz}$ is used for testing in real-time Students must show the RFID card in front of RFID reader and then it is checked for a match in the database of card numbers. Then success 
or failure is reported by displaying it on the LCD. If success, it proceeds to face verification proceeds to face verification process. Face detection is an easy and simple task for humans, but not for computers .It has been regarded as the most complex and challenging problems in the field of computer vision due to large intra-class variations caused by the charges in facial appearance, lighting and expression. Such variations result in the face distribution to be highly nonlinear and complex in any space that is linear to the original image space. Face detection is the process of identifying one or more human faces in images or videos. It plays an important part of in many biometric, security and surveillance systems, as well as image and indexing systems.

\section{SYSTEM DESIGN}

The schematic diagram of the proposed scheme is shown in Fig. 1. The system is designed to first identify the student and verify the respective student biometrically. It increases the security and accuracy of attendance management because of two levels of authentication. RFID Systems has evidences to be the smartest way for student identification. Several biometric verification methods exist like Iris, Face, and Fingerprinted. Face verification robust against different head poses is chosen as the method of biometric verification in the proposed system. RFID System uses the RFID cards to identify the student. Face Verification System uses an individual

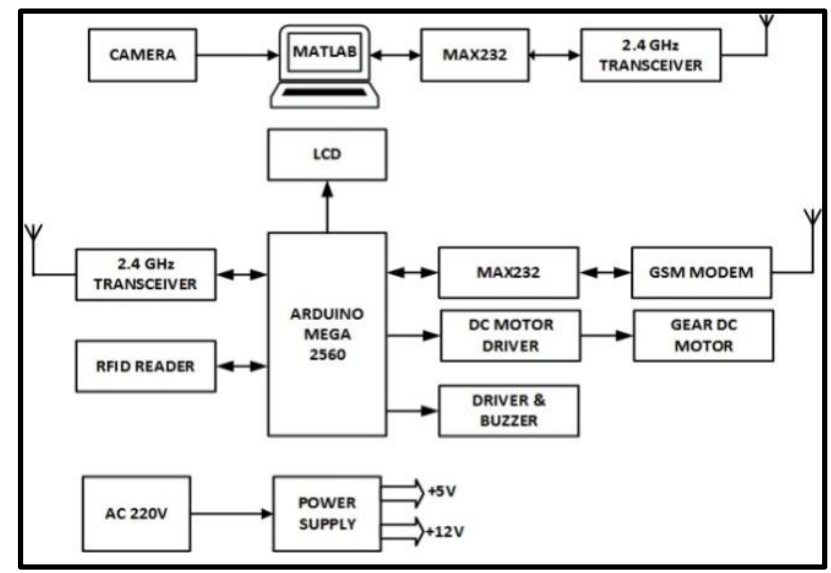

Fig.1. Block Diagram of Complete System

\section{OPERATIONAL CONCEPT}

A. RFID Student Identification System

RFID Student Identification System Every student is provided with an RFID card. RFID Cards are assigned a unique 10 digit RFID card numbers by the manufacturer. The card number is only readable and there forfeit is mapped to their respective student roll number in a RFIDROLL database. Low frequency $(125 \mathrm{KHz}) \mathrm{RFID}$ card is used which could only be detected at a range of $5 \mathrm{~cm}$ from the RFID reader. Thus, collisions of RFID cards are avoided. Smart RFID Proximity Contactless Card Reader $125 \mathrm{KHz}$ is used for testing in real-time Students must show the RFID card in front of RFID reader and then it is checked for a match in the database of card numbers. Then success or failure is reported. If success, it proceeds to face verification process else RFID card number saved in log file ad it waits for another RFID user.

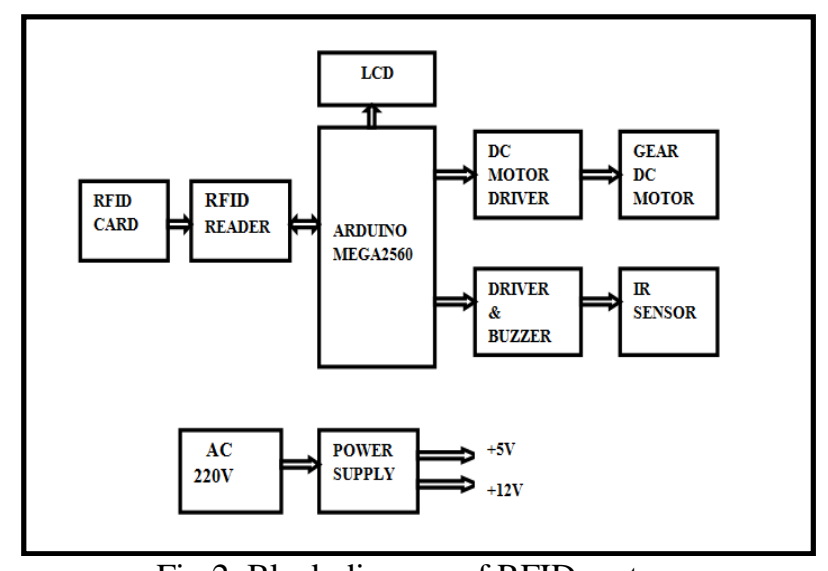

Fig.2. Block diagram of RFID system 


\section{B. Face recognition}

Face recognition is a really challenging problem and it is intrinsically designed to deliver the best performance possible in different head poses conditions.. Each FANNC classifier is trained by the set of face images of the corresponding student taken under different head pose conditions

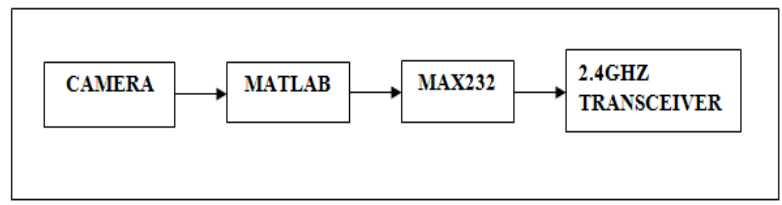

Fig. 3 Block Diagram of face recognition system

Face recognition System uses an individual to verify the face of each student exclusively as shown in Fig. 3 once the face recognition is cleared the ok signal from the system is sent via $2.4 \mathrm{Ghz}$ transceiver to the microcontroller Atmega 2560 which is a part of Arduino mega2560.MATLAB computer vision system tool used to identify various parts of human faces like nose, face, eyes etc. This MATLAB tool is based on viola Jones face detection algorithm .After applying different threshold values various parts of face is identified successfully for different images containing on to multiple faces in it

C. overall hardware implementation

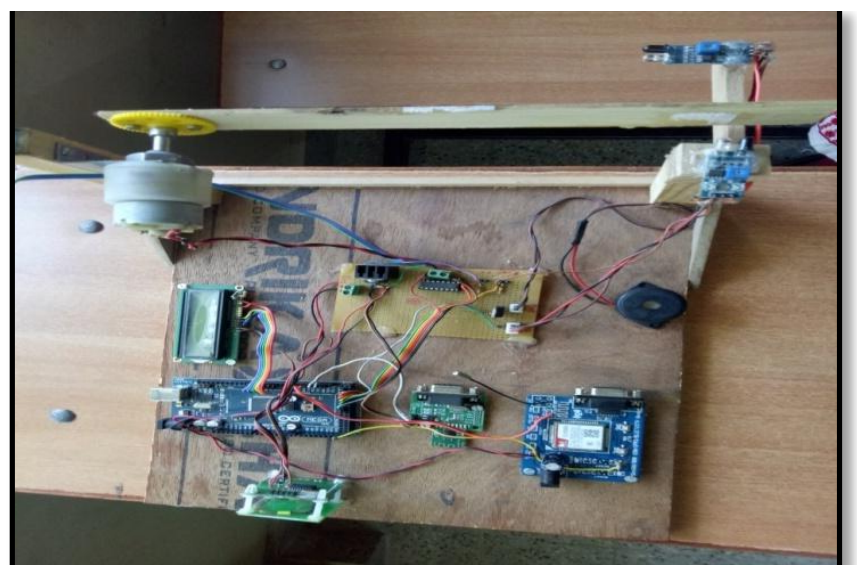

Fig.3. Hardware Part of RFID System

Radio-frequency identification (RFID) is the use of a wireless non-contact system that uses radio-frequency electromagnetic fields to transfer data from a tag attached to an object, for the purposes of automatic identification and tracking. Some tags require no battery and are powered and read at short ranges via magnetic fields (electromagnetic induction). Others use a local power source and emit radio waves (electromagnetic radiation at radio frequencies). The tag contains electronically stored information which may be read from up to several meters away. Unlike a barcode, the tag does not need to be within line of sight of the reader and may be embedded in the tracked object.

RFID tags are used in many industries. An RFID tag attached to an automobile during production can be used to track its progress through the assembly line. Pharmaceuticals can be tracked through warehouses. Livestock and pets may have tags injected, allowing positive identification of the animal.

\section{FACE RECOGNITION}

Face recognition System uses an individual to verify the face of each student. Face detection is the process of identifying one or more human faces in images or videos.In order to make the system work in real time, we need to have 3 components. The three components are face detection, face tracking, and then lastly face recognition. Those components will be described in detail in the next few sub topics, but we need to figure out why we need those three first.

The 3 components necessary for Face detection

For face detection, it is quite obvious, because if a face cannot be detected, how can it be recognized. For face tracking, it is required because real time analysis is done. In Imaging wehave 30 frames per second, if same people cannot be tracked it is not possible to analyze 30 frames per second for finding a face on one computer. This is simply wasting 
calculation power and therefore not efficient. Hence, face tracking is necessary for avoiding unnecessary calculations. For face recognition, it is required because the goal of the project is recognizing the face. By comparing the key elements of the face to the faces stored in the database, we will be able to recognize one person.

\section{a. Face detection}

Face detection is the process of identifying one or more human faces in images or videos.

Algorithm description: For this project vision. Cascade Object Detector System object in vision toolbox of Matlab 2013a to detect people's face using Viola-Jones algorithm. Viola-Jones algorithm is an examining box for a sliding window in an image to try to match different dark/light regions so it can identify a face. The size of the window varies on different scales for different faces, however, the ratio of the window remains unchanged.

\section{b. Face Tracking}

Algorithm description: We are using Kanade-Lucas-Tomasi KLT algorithm to do face tracking. This algorithm is basically based on feature point tracking on the first face, and keeps on tracking it until there is no feature point available.

For the first feature points set on tracking, we used Eigen value algorithm to find corner points. Basically this is ShiTomasi corner detection algorithm which detects the corner. It directly computes the value of Eigen values to determine whether it is a point of interest or not.

After detecting those points, we will be able to track each of the points we found from Shi-Tomasi corner detection. For each consecutive frame we will try to match the points from the step above. There might be points missing, and if it is the case we will rule them out. As long as there are at least 2 points exist in the video frame, we will be able to continue tracking the face by finding out the affine transformation of those points. Then because we are running in real-time, we will want the corner detection algorithm to run again once all corner points are gone.

\section{c. Face recognition}

Algorithm description:We used Principal Component Analysis to find a match in the database. This technique is also used in image compression. It is a way of identifying patterns in data, and expresses the data in the way that highlight their similarities and difference. Basically, first we need a set of data images we store in our program. After we have used PCA on the database, the original data will be in the forms of the eigenvectors we found from the correlation matrix (this is interesting here. Correlation matrix standardized the data). After inputting an image, we will measure the difference between the eigenvectors in input images with the original images in the dataset, and then we need to determine which picture has the least difference to identify the input image. One last thing to note, we used face detection in the first step to help create our database in real time.

\section{RESULTS AND DISCUSSION}

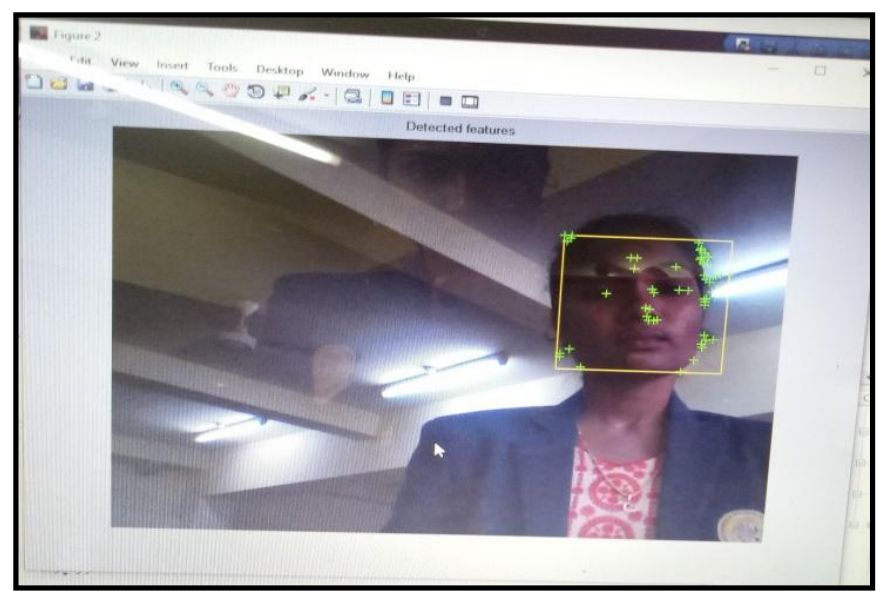

Fig 4: Face detection

For face detection, our program have $>90 \%$ rate on detecting the frontal face, However, there are false positives exist, and to further reduce the false positives we need a stronger classifier. To break this face detection algorithm, a minor issue is to paint a balloon or hold a photo to make the system detect the faces. Those false positives are hard to clean, however, we can integrate other methods to improve the quality like integrating a skin color test. Another way to break it is through painting the face. If you paint your face so the dark/light pattern becomes less recognizable, the algorithm fails. This is understandable because even for our human, it is hard to recognize someone with their face painted. 


\section{RESULT OF TRACKING}

Tracking is working really well on a single target. While rotating the face we can see some corner points missing, and that the polygon window will continue move with our movement when we prevented face detection from running in every frame. The result shows that face detection only needs to be executed when losing the target, and therefore this speeds up our face recognition system.

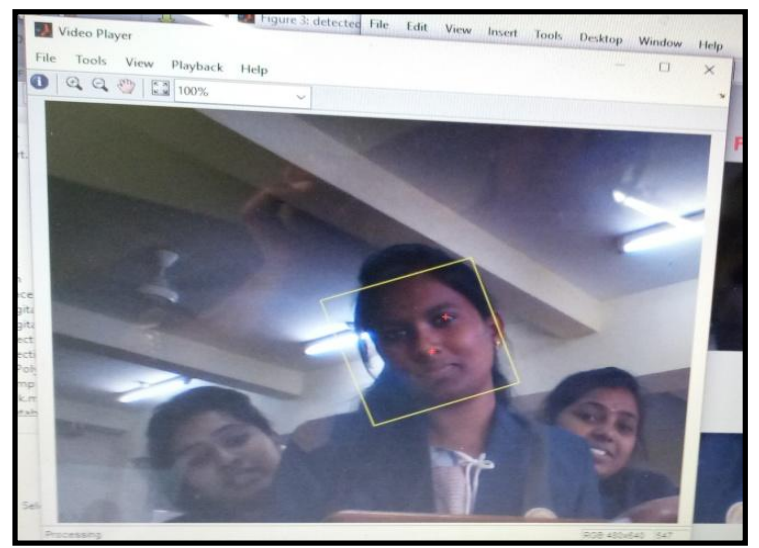

Fig.5.Face Tracking Image

However, we need tracking not only for tracking the same person, reducing the run time of the program; we also need to identify the person's movements in the future. To break the face tracking program, one just need to make a false positive face detection, and then the tracking algorithm will break because the face detection failed. Another way to break the system is through lightning. The system lose track of a face if under extreme light circumstances like strong light or very dim light. But still, comparing this with human tracking system, we would not be able to track a person under such circumstance after all.

\section{RESULT OF RECOGNITION}

The recognition rate of our program is about $80 \%$ due to the similarities of girls with long hair which covers their identities. Note, this recognition rate does not include the rate of failing the face detection. Therefore, the total success rate for our program is lower. The face recognition system did not do anything for long rage objects, and for those smaller faces we simply stretch them to make them go under the same procedure as described in the design phase.

Therefore, to break the program, you just have to stand really far away from the camera during training, and then let two girls stand really far away to test our database. The results will confuse the computer.

\section{RFID Results}

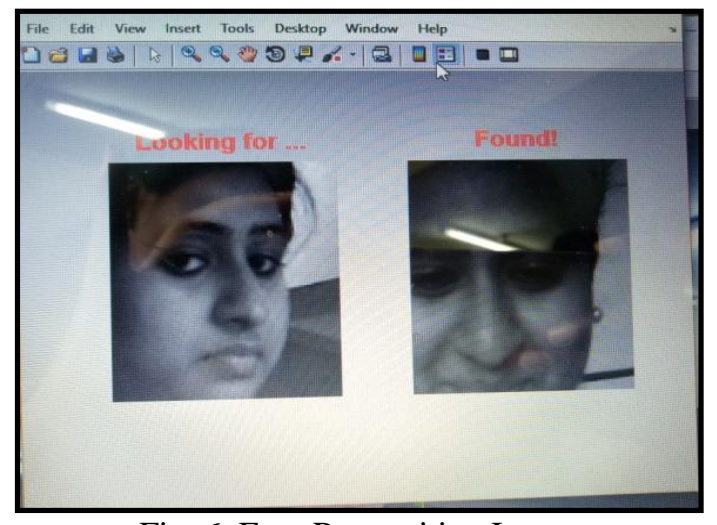

Fig. 6 .Face Recognition Image

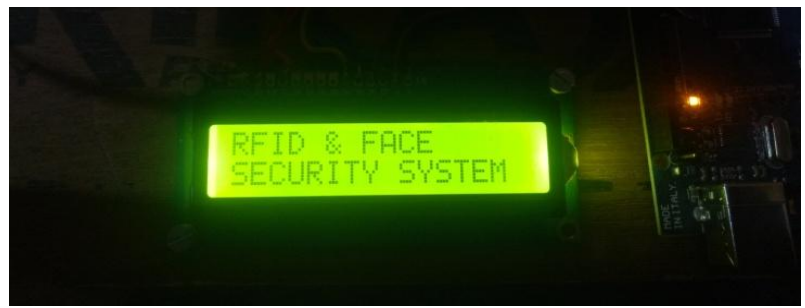




\section{IJARCCE}

\section{CONCLUSIONS}

A compact and reliable automated gate control system is proposed using RFID and Face recognition technology which supports student's identification and verification. The system is computationally faster. The system is secure from proxy gate. The system can be implemented easily using Webcam, RFID reader and Computer of the already existing system. Future work will be focused on testing the system under varied illumination conditions and face verification system with confidence removes the dependency on RFID student identification system.

\section{FUTURE WORK}

To improve the rate of face detection, we need to combine face detection and skin tone mapping together to make better classifiers. Viola-Johns algorithm will only ensure we have the object with the correct shading, but it does not necessarily be human. Therefore to improve the result, a human skin classifier is necessary. For those human skin tone classifiers, we need sample collected from all over the world because the skin tones are all different.

What to improve on feature detection

Right now we are only using corner detection from the area that we are interested in. This is not entirely accurate due to different lighting conditions and different scales of the faces. In the future, if we have a programmable camera, we will program the camera to get the database sample with the same scale as the real test objects.

\section{REFERENCES}

[1] O. Shoewu and O.A. Idowu, "Development of Attendance Management System using Biometrics" The Pacific Journal of Science and Technology, vol 13. No 1. May 2012.

[2] Murizah Kassim, Hasbullah Mazlan, Norliza Zaini, Muhammad Khidhir Salleh, "Web-based Student Attendance System using RFID Technology” 2012 IEEE Control and System Graduate ResearchColloquium, July 16 - 17, UiTM, Shah Alam, Malaysia.

[3] O. Shoewu, O.M. Olaniyi, A. Lawson, "Embedded Computer Computer Computer-Based Lecture Attendance Based Lecture Attendance Management System” Afr J Comp \& ICT,Vol 4. No. 3, 2011

[4] Naveed Khan Balcoh, M. Haroon Yousaf, Waqar Ahmad and M. Iram Baig,“Algorithm for Efficient Attendance Management: Face Recognition based approach" IJCSI International Journal of Computer Science Issues, Vol. 9, Issue 4, No 1, July 2012

[5] Yohei KAWAGUCHI ,Tetsuo SHOJI ,Weijane LIN, and Koh KAKUSHO "Face Recognition-based Lecture Attendance System”, Department of Intelligence Science and Technology, Graduate School of Informatics, Kyoto University.

[7] Mr. C. S. P a til, Mr. R. R. Karhe, Mr. M. D. Jain, Student Attendance System and Authentication using Face Recognition, International Journal of Engineering Research \& Technology (IJERT), Vol. 3 Issue 7, July - 2014

[8] M.N.Shah Zainudin., Radi H.R., S.Muniroh Abdullah., Rosman Abd.Rahim., M.Muzafar Ismail," Face Recognition using PCA and LDA", International Journal of Electrical \& Computer Sciences

[9] Z.-H. Zhou, S. Chen, and Z. Chen. FANNC: a fast adaptive neural network classifier. Knowledge and Information Systems, 2000, vol.2, no.1, pp.115-129

[10] W. ZHAO, R. CHELLAPPA, P. J. PHILLIPS, A. ROSENFELD. Face recognition: A literature survey 2003.

[11]Chintalapati, S, Raghunadh, M.V.Automated attendance management system based on face recognition algorithms. Computational Intelligence and Computing Research (ICCIC), 2013

[12] Changxing Ding, Dacheng Tao. A Comprehensive Survey on Pose-Invariant Face Recognition, CVPR July 2015

[13] Huy Tho Ho, Rama Chellappa. Pose-Invariant Face Recognition Using Markov Random Fields. IEEE Transactions on Image Processing, vol. 22, No. 4, April 2013

[14] Ralph Gross, Simon Baker, Iain Matthews, and Takeo Kanade, Face Recognition across Pose and Illumination. - Springer Handbook of face recognition, 2011. 